Research Article

\title{
Second-Order Nonlinear Impulsive Integro-Differential Equations of Mixed Type and Optimal Controls in Fractional Power Spaces
}

\author{
Y. Peng \\ Department of Mathematics, Guizhou University, Guiyang, Guizhou 550025, China \\ Correspondence should be addressed to Y. Peng, pengyf0803@163.com
}

Received 23 September 2010; Revised 20 November 2010; Accepted 27 November 2010

Academic Editor: Irena Lasiecka

Copyright (C) 2010 Y. Peng. This is an open access article distributed under the Creative Commons Attribution License, which permits unrestricted use, distribution, and reproduction in any medium, provided the original work is properly cited.

A class of second-order nonlinear impulsive integro-differential equations of mixed type whose principal part is given by time-varying generating operators in fractional power spaces is considered. We introduce the reasonable PC- $\alpha$-mild solution of second-order nonlinear impulsive integro-differential equations of mixed type and prove its existence. The existence of optimal controls for a Lagrange problem of systems governed by second-order nonlinear impulsive integro-equations of mixed type is also presented. An example is given for demonstration.

\section{Introduction}

Some interesting models of mathematical biology or population, mechanics of materials, nuclear physics, and so forth, can be written in terms of second-order nonlinear partial integro-differential equations. This is the case of the model proposed to describe viscoelastic problems with memory. The system is given by

$$
\begin{aligned}
\ddot{x}(t)+A(t) \dot{x}(t) & =f(t, x(t), \dot{x}(t),(G x)(t),(H x)(t)), \quad t \in(0, T] \backslash \Theta, \\
x(0) & =x_{0}, \quad \Delta x\left(t_{i}\right)=J_{i}^{0}\left(x\left(t_{i}\right), \dot{x}\left(t_{i}\right)\right), \quad t_{i} \in \Theta, \\
\dot{x}(0) & =x_{1}, \quad \Delta \dot{x}\left(t_{i}\right)=J_{i}^{1}\left(x\left(t_{i}\right), \dot{x}\left(t_{i}\right)\right), \quad t_{i} \in \Theta,
\end{aligned}
$$

where $\{A(t) \mid 0 \leq t \leq T\}$ generates an evolution system $\{U(t, s) \mid 0 \leq s \leq t \leq T\}$ in the parabolic case in Banach spaces $X$ (see [1-3]). G, $H$ are nonlinear integral operators given by

$$
(G x)(t)=\int_{0}^{t} k(t, \tau) g(\tau, x(\tau), \dot{x}(\tau)) d \tau, \quad(H x)(t)=\int_{0}^{T} m(t, \tau) h(\tau, x(\tau), \dot{x}(\tau)) d \tau .
$$


$\Theta=\left\{t_{i} \in(0, T) \mid 0=t_{0}<t_{1}<\cdots<t_{n}<t_{n+1}=T\right\}, J_{i}^{0}, J_{i}^{1}(i=1,2, \ldots, n)$ are nonlinear maps, and $\Delta x\left(t_{i}\right)=x\left(t_{i}+0\right)-x\left(t_{i}\right), \Delta \dot{x}\left(t_{i}\right)=\dot{x}\left(t_{i}+0\right)-\dot{x}\left(t_{i}\right)$. This represents the jump in the state $x$, $\dot{x}$ at time $t_{i}$, respectively, with $J_{i}^{0}, J_{i}^{1}$ determining the size of the jump at time $t_{i}$.

In fact, since the end of last century, impulsive evolution equations on infinitedimensional spaces have been investigated by many authors including us. Particularly, Ahmed and we considered optimal control problems of systems governed by first-order impulsive evolution equations and first-order impulsive integro-differential equations [4-7]. Recently, we discussed the second-order impulsive evolution equations and the second-order impulsive integro-differential equations and their optimal controls in general Banach spaces [8-11]. In addition, to our knowledge, the second-order impulsive functional differential equations and the second-order impulsive integro-differential equations whose principal operator is bounded have been deeply studied by many authors [12-16]. However, the second-order impulsive integro-differential evolutions equations of mixed type whose principle operator is unbounded in infinite dimensional fractional power spaces and corresponding optimal control problems have not been extensively considered in the literature.

Reducing the second-order evolution equations to the first-order evolution equations, we introduce a family of unbounded linear matrix operators $\mathfrak{A}(t), 0 \leq t \leq T$ and prove that $\{\mathfrak{A}(t) \mid 0 \leq t \leq T\}$ generates an evolution system $\{\bar{U}(t, s) \mid 0 \leq s \leq t \leq T\}$ which can be represented by $U(t, s), 0 \leq s \leq t \leq T$. Based on the evolution system $\{\bar{U}(\cdot, \cdot)\}$, we introduce a reasonable PC- $\alpha$-mild solution of (1.1). Using the interpolation space technique, we can overcome the difficulty brought by fractional power spaces. Next, by a virtue of the generalized Gronwall lemma with singularity, impulse, and integrals of mixed type given by us, one can overcome the difficulty brought by operator $H$ to get a priori estimate of PC- $\alpha$-mild solution. By compactness condition of space $\mathrm{PC}^{1}\left([0, T], X_{\alpha}\right)$ and Leray-Schauder fixed point theory, we can obtain the existence of PC- $\alpha$-mild solution for (1.1). Particularly, introducing new norm, we use the contraction mapping principle to give the uniqueness of $\alpha$-mild solution for the second-order nonlinear integro-differential equation (1.1) without impulses (see Remark 3.4). A Lagrange problem of a system governed by (1.1) whose cost functional includes both $x$ and $\dot{x}$ is investigated. By the structure of $\{\bar{U}(\cdot, \cdot)\}$ and compactness of $\{U(\cdot, \cdot)\}$, the existence of optimal controls is verified.

The rest of the paper is organized as follows. In Section 2, we give some associated notations and important lemmas. In Section 3, the existence of PC- $\alpha$-mild solution for (1.1) is presented. In Section 4, we consider a Lagrange problem of system governed by (1.1) and prove the existence of optimal controls. At last, an example demonstrates the applicability of our results.

\section{Preliminaries}

Let $X, Y$ denote a pair of Banach spaces. If $X$ is continuously embedded in $Y$, we write $X \hookrightarrow Y$; if $X$ is compactly embedded in $Y$, we write $X \hookrightarrow \hookrightarrow Y$. Set $\bar{\Delta}=\{(t, s) \mid 0 \leq s \leq t \leq T\}, £(X)$ is the class of (not necessarily bounded) linear operators in $X . £_{b}(X)$ stands for the family of bounded linear operators in $X$. For $A \in £(X)$, let $\rho(A)$ denote the resolvent set and $R(\lambda, A)$ the resolvent corresponding to $\lambda \in \rho(A)$.

Assumption $A$. $\left(\mathrm{P}_{1}\right)$ Let $\{A(t) \mid t \in[0, T]\}$ be a family of closed linear operators in $X$, the domain $D(A(t))=D$ of $A(t)$, and $t \in[0, T]$ dense in $X$ and independent of $t$. 
$\left(\mathrm{P}_{2}\right)$ For $t \in[0, T]$, the resolvent $R(\lambda, A(t))$ of $A(t)$ exists for all $\lambda$ with $\operatorname{Re} \lambda \leq 0$ and there exists a constant $M>0$ such that

$$
\|R(\lambda, A(t))\|_{E(X)} \leq \frac{M}{|\lambda|+1}, \quad \text { for } t \in[0, T] .
$$

$\left(\mathrm{P}_{3}\right)$ There exist constants $L>0$ and $0<\alpha \leq 1$ such that

$$
\left\|(A(t)-A(s)) A^{-1}(\tau)\right\|_{E(X)} \leq L|t-s|^{\alpha} \quad \text { for } s, t, \tau \in[0, T] .
$$

Let $X_{1}=\left\{D,\|\cdot\|_{1}\right\}$ where $\|x\|_{1}=\|A x\| . X_{1}$ is a Banach space and $X_{1} \hookrightarrow X$. More generally, in a usual way we introduce the fractional power operator $A^{\alpha}(t)(\alpha \in(0,1))$, which satisfies $D(A)=D(A(t))$ and $D\left(A^{\alpha}\right)=D\left(A^{\alpha}(t)\right)$ for $t \in[0, T]$. Let $\|x\|_{\alpha}=\left\|A^{\alpha} x\right\|$ for $x \in$ $D\left(A^{\alpha}(t)\right)$ and denote the Banach space $\left\{D\left(A^{\alpha}\right),\|\cdot\|_{\alpha}\right\}$ as $X_{\alpha}$. Then it is clear that $X_{\beta} \hookrightarrow X_{\alpha}$ for $0 \leq \alpha \leq \beta \leq 1$. Define $\mathrm{PC}\left([0, T], X_{\alpha}\right) \equiv\left\{x:[0, T] \rightarrow X_{\alpha} \mid x\right.$ as continuous at $t \in$ $[0, T] \backslash \Theta ; x$ is continuous from left and has right-hand limits at $t \in \Theta\}, \operatorname{PC}^{1}\left([0, T], X_{\alpha}\right) \equiv\{x \in$ $\left.\mathrm{PC}\left([0, T], X_{\alpha}\right) \mid \dot{x} \in \operatorname{PC}([0, T], X)\right\}$. It can be seen that $\mathrm{PC}\left([0, T], X_{\alpha}\right)$ and $\mathrm{PC}^{1}\left([0, T], X_{\alpha}\right)$ are Banach spaces, respectively, with the norms

$$
\|x\|_{\mathrm{PC}}=\max \left\{\sup _{t \in[0, T]}\|x(t+0)\|_{\alpha} \sup _{t \in[0, T]}\|x(t-0)\|_{\alpha}\right\}, \quad\|x\|_{\mathrm{PC}^{1}}=\|x\|_{\mathrm{PC}}+\|\dot{x}\|_{\mathrm{PC}} .
$$

For the initial value problem

$$
\begin{aligned}
\dot{x}(t)+A(t) x(t) & =0, \quad t \in(0, T], \\
x(0) & =x_{0},
\end{aligned}
$$

it is well known that (2.4) has a unique classical solution $x$. Moreover, $x \in C^{1}([0, T], X)$ provided $x_{0} \in D(A)$. Further, there exists a unique evolution operator $U(t, s) \in L_{b}(X),(t, s) \in$ $\bar{\Delta}$, such that every solution of (2.4) can be represented in the form

$$
x(t)=U(t, 0) x_{0} .
$$

Consider the following second-order initial value problem:

$$
\begin{gathered}
\ddot{x}(t)+A(s) \dot{x}(t)=0, \quad t \in(0, T], \\
x(0)=x_{0}, \quad \dot{x}(0)=x_{1} .
\end{gathered}
$$

Set $v(t)=\left(\begin{array}{c}x(t) \\ \dot{x}(t)\end{array}\right)$ and $v_{0}=\left(\begin{array}{c}x_{0} \\ x_{1}\end{array}\right) ;(2.6)$ can be rewritten as

$$
\begin{aligned}
\dot{v}(t)+\mathfrak{A}(t) v(t) & =0, \quad t \in(0, T], \\
v(0) & =v_{0},
\end{aligned}
$$

where $\mathfrak{A}(t)=\left(\begin{array}{cc}0 & -I \\ 0 & A(t)\end{array}\right)$. By $[9$, Theorem 2.A], we have the following theorem. 
Theorem 2.1. Under the Assumption $A$, (2.7) has a unique evolution system $\{\bar{U}(t, s) \mid(t, s) \in \bar{\Delta}\}$ given by

$$
\bar{U}(t, s)=\left(\begin{array}{cc}
I & \int_{s}^{t} U(\tau, s) d \tau \\
0 & U(t, s)
\end{array}\right)
$$

In order to derive a priori estimates on the PC- $\alpha$-mild solution of integro-differential equation of mixed type, we need the following generalized Gronwall inequality with impulses.

Theorem 2.2. Let $x \in P C\left([0, T], X_{\alpha}\right)$ and satisfy the following inequality:

$\|x(t)\|_{\alpha} \leq a+b \int_{0}^{t}|t-\tau|^{-\gamma}\|x(\tau)\|_{\alpha} d \tau+c \int_{0}^{t}|t-\tau|^{-\gamma}\left\|x_{\tau}\right\|_{B} d \tau+\delta \int_{0}^{T}\|x(\tau)\|_{\alpha}^{\lambda} d \tau+\sum_{0<t_{k}<t} \theta_{k}\left\|x\left(t_{k}\right)\right\|_{\alpha}$

for all $t \in[0, T]$, where $1>\lambda, \gamma>0$, and $a, b, c, \delta, \theta_{k} \geq 0$ are constants, and $\left\|x_{t}\right\|_{B}=$ $\sup _{0 \leq s \leq t}\|x(s)\|_{\alpha}$. Then there exists constant $M>0$ such that

$$
\|x(t)\|_{\alpha} \leq M, \quad \forall t \in[0, T]
$$

Proof. By the inequality (2.9) and [7, Lemma 2.1], there exist $a_{0}>0, b_{0}>0$ such that

$$
\left\|x_{t}\right\|_{B} \leq a_{0}\left(a+\delta \int_{0}^{T}\|x(\tau)\|_{\alpha}^{\lambda} d \tau\right)+b_{0} \int_{0}^{t}\left\|x_{\tau}\right\|_{B} d \tau+\sum_{0<t_{k}<t} a_{0} \theta_{k}\left\|x\left(t_{k}\right)\right\|_{B} .
$$

By [17, Lemma 1.7.1], we have

$$
\begin{aligned}
\left\|x_{t}\right\|_{B} & \leq a_{0}\left(a+\delta \int_{0}^{T}\|x(\tau)\|_{\alpha}^{\lambda} d \tau\right) \prod_{0<t_{k}<t}\left(1+a_{0} \theta_{k}\right) e^{b_{0} t} \\
& \leq a_{0}\left(a+\delta \int_{0}^{T}\|x(\tau)\|_{\alpha}^{\lambda} d \tau\right) \prod_{0<t_{k}<T}\left(1+a_{0} \theta_{k}\right) e^{b_{0} T} .
\end{aligned}
$$

Using the argument method [10, Lemma 3.1], there exists constant $M>0$ such that

$$
\|x(t)\|_{\alpha} \leq M, \quad \forall t \in[0, T]
$$

This completes the proof.

Next, we extend the Ascoli-Arzela Theorem from $C([0, T], X)$ to $\mathrm{PC}^{1}\left([0, T], \mathrm{X}_{\alpha}\right)$. 
Theorem 2.3. Suppose $\mathfrak{W} \subset P C^{1}\left([0, T], X_{\alpha}\right)$ is a subset. If the following conditions are satisfied:

(1) $\mathcal{W}$ is uniformly bounded subset of $P C^{1}\left([0, T], X_{\alpha}\right)$,

(2) $W$ is equicontinuous in $\left(t_{i}, t_{i+1}\right), i=0,1,2, \ldots, n$, where $t_{0}=0, t_{n+1}=T$,

(3) its $t$-sections $\mathcal{W}(t) \equiv\{x(t) \mid x \in \mathcal{W}, t \in[0, T] \backslash \Theta\}, \dot{\mathcal{W}}(t) \equiv\{\dot{x}(t) \mid x \in \mathcal{W}, t \in$ $[0, T] \backslash \Theta\}, \mathcal{W}\left(t_{i}+0\right) \equiv\left\{x\left(t_{i}+0\right) \mid x \in W\right\}, \mathcal{W}\left(t_{i}-0\right) \equiv\left\{x\left(t_{i}-0\right) \mid x \in \mathcal{W}\right\}$, $\dot{\mathcal{W}}\left(t_{i}+0\right) \equiv\left\{\dot{x}\left(t_{i}+0\right) \mid x \in W\right\}$, and $\dot{\mathcal{W}}\left(t_{i}-0\right) \equiv\left\{\dot{x}\left(t_{i}-0\right) \mid x \in \mathcal{W}\right\}$ are relatively compact subsets of $X_{\alpha}$,

then $W$ is a relatively compact subset of $P C^{1}\left([0, T], X_{\alpha}\right)$.

Proof. Let $W=x(\cdot) \subset \operatorname{PC}^{1}\left([0, T], X_{\alpha}\right)$ satisfy assumptions of Theorem 2.3 and $\left\{x_{m}(\cdot)\right\}$ any sequence of $W$. Define $W^{1}=W_{\left[0, t_{1}-\right]}=\left\{x^{1} \in C^{1}\left(\left[0, t_{1}\right], X\right) \mid x^{1}(t)=x(t)\right.$ for $t \in$ $\left.\left[0, t_{1}\right), x^{1}\left(t_{1}\right)=x\left(t_{1}-0\right), x \in W\right\}$. By Ascoli-Arzela Theorem, $W^{1}$ is a relatively compact subset of $C^{1}\left(\left[0, t_{1}\right], X_{\alpha}\right)$. Then, there exists a subsequence of $\left\{x_{m}\right\}$, labeled $\left\{x_{m}^{1}\right\} \subset W$, and $x^{1} \in C^{1}\left(\left[0, t_{1}\right], X_{\alpha}\right)$, such that

$$
x_{m}^{1} \longrightarrow x^{1} \quad \text { in } C^{1}\left(\left[0, t_{1}\right], X\right) \text { as } m \longrightarrow+\infty \text {. }
$$

Define $W^{2}=W_{\left[t_{1}+t_{2}-\right]}=\left\{x^{2} \in C^{1}\left(\left[t_{1}, t_{2}\right], X\right) \mid x^{2}(t)=x(t)\right.$ for $t \in\left(t_{1}, t_{2}\right)$ and $x^{2}\left(t_{1}\right)=x\left(t_{1}+\right.$ $\left.0), x^{2}\left(t_{2}\right)=x\left(t_{2}-0\right), x \in W\right\}$. It is not difficult to see that, due to Ascoli-Arzela Theorem again, $W^{2}$ is a relatively compact subset of $C^{1}\left(\left[t_{1}, t_{2}\right], X_{\alpha}\right)$. There exists a subsequence of $\left\{x_{m}^{1}\right\}$, labeled $\left\{x_{m}^{2}\right\} \subset W$, and $x^{2} \in C^{1}\left(\left[t_{1}, t_{2}\right], X_{\alpha}\right)$ such that

$$
x_{m}^{2} \longrightarrow x^{2} \text { in } C^{1}\left(\left[t_{1}, t_{2}\right], X\right) \text { as } m \longrightarrow+\infty .
$$

In general, define $W^{i}=W_{\left[t_{i}+, t_{i+1}-\right]}=\left\{x^{i} \in C^{1}\left(\left[t_{i}, t_{i+1}\right], X\right) \mid x^{2}(t)=x(t)\right.$ for $t \in$ $\left(t_{i}, t_{i+1}\right)$ and $\left.x^{i}\left(t_{i}\right)=x\left(t_{i}+0\right), x^{i}\left(t_{i+1}\right)=x\left(t_{i+1}-0\right), x \in W\right\}(i=2, \ldots, n+1)$. Similarly, $W^{\mathrm{i}}$ is a relatively compact subset of $C^{1}\left(\left[t_{i}, t_{i+1}\right], X_{\alpha}\right)$. There exists a subsequence of $\left\{x_{m}^{i-1}\right\}$, labeled $\left\{x_{m}^{i}\right\} \subset W$, and $x^{i} \in C^{1}\left(\left[t_{i}, t_{i+1}\right], X\right)$ such that

$$
x_{m}^{i} \longrightarrow x^{i} \quad \text { in } C^{1}\left(\left[t_{i}, t_{i+1}\right], X\right) \text { as } m \longrightarrow+\infty .
$$

Setting

$$
x(t)= \begin{cases}x^{1}(t), & t \in\left[0, t_{1}\right], \\ x^{2}(t), & t \in\left(t_{1}, t_{2}\right], \\ \vdots & \\ x^{n+1}(t), & t \in\left(t_{n}, T\right],\end{cases}
$$

then

$$
x_{m}^{n+1} \longrightarrow x \text { in } \operatorname{PC}^{1}\left([0, T], X_{\alpha}\right) \text { as } m \longrightarrow+\infty .
$$

Thus, the set $W$ is a relatively compact set. 


\section{Solution of Second-Order Nonlinear Integro-Differential Equations of Mixed Type}

We introduce reasonable mild solution for (1.1) and show the existence of PC- $\alpha$-mild solution.

Definition 3.1. A function $x \in \mathrm{PC}^{1}\left([0, T], \mathrm{X}_{\alpha}\right)$ is said to be a PC- $\alpha$-mild solution of (1.1) if $x$ satisfies the following integral equation:

$$
\begin{aligned}
x(t)= & x_{0}+\int_{0}^{t} U(\tau, 0) x_{1} d \tau+\int_{0}^{t} \int_{\tau}^{t} U(s, \tau) f(\tau, x(\tau), \dot{x}(\tau),(G x)(\tau),(H x)(\tau)) d s d \tau \\
& +\sum_{0<t_{i}<t} J_{i}^{0}\left(x\left(t_{i}\right), \dot{x}\left(t_{i}\right)\right)+\sum_{0<t_{i}<t} \int_{t_{i}}^{t} U\left(s, t_{i}\right) J_{i}^{1}\left(x\left(t_{i}\right), \dot{x}\left(t_{i}\right)\right) d s .
\end{aligned}
$$

We introduce the following assumptions.

Assumption F. (1) The functions $f:[0, T] \times X_{\alpha} \times X_{\alpha} \times X_{\alpha} \times X_{\alpha} \rightarrow X, g:[0, T] \times X_{\alpha} \times X_{\alpha} \rightarrow X_{\alpha}$, and $h:[0, T] \times X_{\alpha} \times X_{\alpha} \rightarrow X_{\alpha}$ are measurable in $t \in[0, T]$ and locally Lipschitz continuous, that is, for all $x_{1}, x_{2}, y_{1}, y_{2}, z_{1}, z_{2} \in X_{\alpha}$, satisfying $\left\|x_{1}\right\|_{\alpha^{\prime}}\left\|x_{2}\right\|_{\alpha^{\prime}}\left\|y_{1}\right\|_{\alpha^{\prime}}\left\|y_{2}\right\|_{\alpha^{\prime}}\left\|z_{1}\right\|_{\alpha^{\prime}}\left\|z_{2}\right\|_{\alpha^{\prime}}$ $\left\|w_{1}\right\|_{\alpha},\left\|w_{2}\right\|_{\alpha} \leq \rho$, we have

$$
\begin{aligned}
& \left\|f\left(t, x_{1}, y_{1}, z_{1}, w_{1}\right)-f\left(t, x_{2}, y_{2}, z_{2}, w_{2}\right)\right\| \\
& \quad \leq L(\rho)\left(\left\|x_{1}-x_{2}\right\|_{\alpha}+\left\|y_{1}-y_{2}\right\|_{\alpha}+\left\|z_{1}-z_{2}\right\|_{\alpha}+\left\|w_{1}-w_{2}\right\|_{\alpha}\right) \\
& \left\|g\left(t, x_{1}, y_{1}\right)-g\left(t, x_{2}, y_{2}\right)\right\|_{\alpha} \leq L(\rho)\left(\left\|x_{1}-x_{2}\right\|+\left\|y_{1}-y_{2}\right\|\right) \\
& \left\|h\left(t, x_{1}, y_{1}\right)-h\left(t, x_{2}, y_{2}\right)\right\|_{\alpha} \leq L(\rho)\left(\left\|x_{1}-x_{2}\right\|+\left\|y_{1}-y_{2}\right\|\right) .
\end{aligned}
$$

(2) There exist a constant $0<\lambda<1$ and a function $q \in L^{r}([0, T],[0,+\infty))(r>1)$ such that

$$
\begin{gathered}
\|f(t, x, y, z, w)\| \leq q(t)\left(1+\|x\|_{\alpha}+\|y\|_{\alpha}+\|z\|_{\alpha}+\|w\|_{\alpha}\right), \quad \forall x, y, z, w \in X_{\alpha} \\
\|g(t, x, y)\|_{\alpha} \leq q(t)\left(1+\|x\|_{\alpha}+\|y\|_{\alpha}\right), \quad \forall x, y \in X_{\alpha} \\
\|h(t, x, y)\|_{\alpha} \leq q(t)\left(1+\|x\|_{\alpha}^{\lambda}+\|y\|_{\alpha}^{\curlywedge}\right), \quad \forall x, y \in X_{\alpha} .
\end{gathered}
$$

(3) $k, m \in C\left([0, T]^{2}, R\right)$.

Assumption J. There exists a constant $b \geq 0$ such that maps $J_{i}^{0}: X_{\alpha} \times X_{\alpha} \rightarrow X_{\alpha}$ and $J_{i}^{1}$ : $X_{\alpha} \times X_{\alpha} \rightarrow X_{\beta_{i}}\left(0<\alpha<\beta_{i}<1\right)$ satisfy

$$
\begin{aligned}
& \left\|J_{i}^{0}\left(x_{1}, y_{1}\right)-J_{i}^{0}\left(x_{2}, y_{2}\right)\right\|_{\alpha} \leq b\left(\left\|x_{1}-x_{2}\right\|_{\alpha}+\left\|y_{1}-y_{2}\right\|_{\beta_{i}}\right) \quad(i=1,2, \ldots, n) \\
& \left\|J_{i}^{1}\left(x_{1}, y_{1}\right)-J_{i}^{1}\left(x_{2}, y_{2}\right)\right\|_{\beta_{i}} \leq b\left(\left\|x_{1}-x_{2}\right\|_{\alpha}+\left\|y_{1}-y_{2}\right\|_{\beta_{i}}\right) \quad(i=1,2, \ldots, n)
\end{aligned}
$$


Theorem 3.2. Suppose that $A(0)$ has a compact resolvent, $\left(x_{0}, x_{1}\right) \in X_{\alpha} \times X_{\beta}, 0<\alpha<\beta \leq$ 1 , and $(\alpha+\beta) / 2<(r-1) / r$. Under the Assumptions $A, F$ and J, the second-order impulsive integrodifferential equation (1.1) has a $P C$ - $\alpha$-mild solution $x \in P C^{1}\left([0, T], X_{\alpha}\right)$.

Proof. Let $\left(x_{0}, x_{1}\right) \in X_{\alpha} \times X_{\beta}$ be fixed; define the operator $P$ on $\mathrm{PC}^{1}\left([0, T], X_{\alpha}\right)$ given by

$$
\begin{aligned}
(P x)(t)= & x_{0}+\int_{0}^{t} U(\tau, 0) x_{1} d \tau+\int_{0}^{t} \int_{\tau}^{t} U(s, \tau) f(\tau, x(\tau), \dot{x}(\tau),(G x)(\tau),(H x)(\tau)) d s d \tau \\
& +\sum_{0<t_{i}<t} J_{i}^{0}\left(x\left(t_{i}\right), \dot{x}\left(t_{i}\right)\right)+\sum_{0<t_{i}<t} \int_{t_{i}}^{t} U\left(s, t_{i}\right) J_{i}^{1}\left(x\left(t_{i}\right), \dot{x}\left(t_{i}\right)\right) d s .
\end{aligned}
$$

By virtue of the properties of evolution system $U(\cdot, \cdot)$ and Assumption J, for $t \in[0, T] \backslash \Theta$, we have $(P x)(t) \in X_{\alpha}$ and

$$
\begin{aligned}
\frac{d}{d t}(P x)(t)= & U(t, 0) x_{1}+\int_{0}^{t} U(t, \tau) f(\tau, x(\tau), \dot{x}(\tau),(G x)(\tau),(H x)(\tau)) d \tau \\
& +\sum_{0<t_{i}<t} U\left(t, t_{i}\right) J_{i}^{1}\left(x\left(t_{i}\right), \dot{x}\left(t_{i}\right)\right) .
\end{aligned}
$$

This means that $P x \in \mathrm{PC}^{1}\left([0, T], \mathrm{X}_{\alpha}\right)$ for $x \in \mathrm{PC}^{1}\left([0, T], \mathrm{X}_{\alpha}\right)$.

For $x, y \in \mathrm{PC}^{1}\left([0, T], X_{\alpha}\right)$ and $\|x\|_{\mathrm{PC}^{1}},\|y\|_{\mathrm{PC}^{1}} \leq \rho$ in which $\rho$ is a constant. Using Assumptions $\mathrm{F}(1)$ and $\mathrm{J}$, we have

$$
\begin{aligned}
& \|(P x)(t)-(P y)(t)\|_{\alpha}+\left\|\frac{d}{d t}(P x)(t)-\frac{d}{d t}(P y)(t)\right\|_{\alpha} \\
& \leq \int_{0}^{t} \int_{\tau}^{t}\|U(s, \tau)\|_{0, \alpha} \| f(\tau, x(\tau), \dot{x}(\tau),(G x)(\tau),(H x)(\tau)) \\
& \quad-f(\tau, y(\tau), \dot{y}(\tau),(G y)(\tau),(H y)(\tau)) \| d s d \tau \\
& +\int_{0}^{t}\|U(t, \tau)\|_{0, \alpha} \| f(\tau, x(\tau), \dot{x}(\tau),(G x)(\tau),(H x)(\tau)) \\
& \quad+\sum_{0<t_{i}<t}\left\|J_{i}^{0}\left(x\left(t_{i}\right), \dot{x}\left(t_{i}\right)\right)-J_{i}^{0}\left(y\left(t_{i}\right), \dot{y}\left(t_{i}\right)\right)\right\|_{\alpha} \\
& +\sum_{0<t_{i}<t} \int_{t_{i}}^{t}\left\|U\left(s, t_{i}\right)\right\|_{\beta_{i}, \alpha}\left\|J_{i}^{1}\left(x\left(t_{i}\right), \dot{x}\left(t_{i}\right)\right)-J_{i}^{1}\left(y\left(t_{i}\right), \dot{y}\left(t_{i}\right)\right)\right\|_{\beta_{i}} d s \\
& +\sum_{0<t_{i}<t}\left\|U\left(t, t_{i}\right)\right\|_{\beta_{i}, \alpha}\left\|J_{i}^{1}\left(x\left(t_{i}\right), \dot{x}\left(t_{i}\right)\right)-J_{i}^{1}\left(y\left(t_{i}\right), \dot{y}\left(t_{i}\right)\right)\right\|_{\beta_{i}}
\end{aligned}
$$




$$
\begin{aligned}
\leq & C(\alpha, \gamma) L^{2}(\bar{\rho})\|m\| T\|x-y\|_{\mathrm{PC}^{1}} \int_{0}^{t} \int_{\tau}^{t}|s-\tau|^{-\gamma} d s d \tau+C(\alpha, \gamma) L^{2}(\bar{\rho})\|m\| T\|x-y\|_{\mathrm{PC}^{1}} \\
& \times \int_{0}^{t}|t-\tau|^{-\gamma} d \tau d s+C(\alpha, \gamma)(1+L(\bar{\rho}))^{2}(\|k\| T+1) \\
& \times \int_{0}^{t} \int_{\tau}^{t}|s-\tau|^{-\gamma}\left[\|x(\tau)-y(\tau)\|_{\alpha}+\|\dot{x}(\tau)-\dot{y}(\tau)\|_{\alpha}\right] d s d \tau \\
& +C(\alpha, \gamma)(1+L(\bar{\rho}))^{2}(\|m\| T+1) \int_{0}^{t} \int_{\tau}^{t}|s-\tau|^{-\gamma}\left[\left\|(x-y)_{\tau}\right\|_{B}+\left\|(\dot{x}-\dot{y})_{\tau}\right\|_{B}\right] d s d \tau \\
& +C(\alpha, \gamma)(1+L(\bar{\rho}))^{2}(\|k\| T+1) \int_{0}^{t}|t-\tau|^{-\gamma}\left[\|x(\tau)-y(\tau)\|_{\alpha}+\|\dot{x}(\tau)-\dot{y}(\tau)\|_{\alpha}\right] d \tau \\
& +C(\alpha, \gamma)(1+L(\bar{\rho}))^{2}(\|m\| T+1) \int_{0}^{t}|t-\tau|^{-\gamma}\left[\left\|(x-y)_{\tau}\right\|_{B}+\left\|(\dot{x}-\dot{y})_{\tau}\right\|_{B}\right] d \tau \\
& +b \sum_{0<t_{i}<t}\left[\left\|x\left(t_{i}\right)-y\left(t_{i}\right)\right\|_{\alpha}+\left\|\dot{x}\left(t_{i}\right)-\dot{y}\left(t_{i}\right)\right\|_{\alpha}\right]+b(T+1) \\
& \times \sum_{0<t_{i}<t} C\left(\beta_{i}, \alpha\right)\left[\left\|x\left(t_{i}\right)-y\left(t_{i}\right)\right\|_{\alpha}+\left\|\dot{x}\left(t_{i}\right)-\dot{y}\left(t_{i}\right)\right\|_{\alpha}\right] \\
\leq & \bar{M}\|x-y\|_{\mathrm{PC}},
\end{aligned}
$$

where $\alpha<\gamma<1$,

$$
\begin{gathered}
\bar{\rho}=\rho+2\|q\|_{L^{1}}\left(\rho+\rho^{\lambda}+1\right)(\|k\|+\|m\|), \\
\bar{M}=2 C(\alpha, \gamma)(L(\bar{\rho})+1)^{2}(\|k\| T+\|m\| T+1) \frac{T^{1-\gamma}+T^{2-\gamma}}{1-\gamma}+b(T+1) \sum_{i=1}^{n}\left(C\left(\beta_{i}, \alpha\right)+1\right) .
\end{gathered}
$$

Hence $P: \mathrm{PC}^{1}\left([0, T], \mathrm{X}_{\alpha}\right) \rightarrow \mathrm{PC}^{1}\left([0, T], \mathrm{X}_{\alpha}\right)$ is a continuous operator.

Let $X \subseteq \mathrm{PC}^{1}\left([0, T], \mathrm{X}_{\alpha}\right)$ be a bounded subset; there exists a constant $\mu>0$ such that $\|x\|_{\mathrm{PC}^{1}} \leq \mu$ for all $x \in \mathcal{X}$. By Assumption $\mathrm{F}(2)$ and the properties of evolution operators, there exists constant $\omega>0$ such that

$$
\|f(t, x(t), \dot{x}(t),(G x)(t),(H x)(t))\| \leq \omega q(t), \quad \forall x \in \mathcal{X}
$$

and $\mathcal{K}=P \mathcal{X}$ is a bounded subset of $\operatorname{PC}^{1}\left([0, T], X_{\alpha}\right)$. Define

$$
\mathcal{K}(t)=\{(P x)(t) \mid x \in \mathcal{X}\}, \quad \dot{\mathcal{K}}(t)=\left\{\frac{d}{d t}(P x)(t) \mid x \in \mathcal{X}\right\} \quad \text { for } t \in[0, T] .
$$


Clearly, $\mathcal{K}(0)=\left\{x_{0}\right\}$ and $\dot{\mathcal{K}}(0)=\left\{x_{1}\right\}$ are compact, and hence, it is only necessary to consider $t>0$. Since $A^{-1}(0)$ is a compact operator, $X_{\beta} \hookrightarrow \hookrightarrow X_{\alpha}$ for $0 \leq \alpha<\beta \leq 1$. For $0<\varepsilon<t \leq T$, define

$$
\begin{gathered}
\mathcal{K}_{\varepsilon}(t)=\left\{\left(P_{\varepsilon} x\right)(t)=U(t, t-\varepsilon)(P x)(t-\varepsilon) \mid x \in X\right\}, \\
\dot{K}_{\varepsilon}(t)=\left\{\frac{d}{d t}\left(P_{\varepsilon} x\right)(t)=U(t, t-\varepsilon) \frac{d}{d t}(P x)(t-\varepsilon) \mid x \in X\right\} .
\end{gathered}
$$

By the properties of $\{U(t, s) \mid(s, t) \in \bar{\Delta}\}$, one can verify that

$$
\begin{aligned}
& \left\|(P x)(t)-\left(P_{\varepsilon} x\right)(t)\right\|_{\alpha} \\
& \leq\left\|x_{0}-U(t, t-\varepsilon) x_{0}\right\|_{\alpha}+\int_{t-\varepsilon}^{t}\|U(s, 0)\|_{\beta, \alpha}\left\|x_{1}\right\|_{\beta} d s \\
& +\|I-U(t, t-\varepsilon)\|_{(\alpha+\beta) / 2, \alpha} \int_{0}^{t-\varepsilon}\|U(s, 0)\|_{\beta,(\alpha+\beta) / 2}\left\|x_{1}\right\|_{\beta} d s \\
& +\int_{t-\varepsilon}^{t} \int_{\tau}^{t}\|U(s, \tau)\|_{0, \alpha}\|f(\tau, x(\tau), \dot{x}(\tau),(G x)(\tau),(H x)(\tau))\| d s d \tau \\
& +\int_{0}^{t-\varepsilon} \int_{t-\varepsilon}^{t}\|U(s, \tau)\|_{0, \alpha}\|f(\tau, x(\tau), \dot{x}(\tau),(G x)(\tau),(H x)(\tau))\| d s d \tau \\
& +\|I-U(t, t-\varepsilon)\|_{(\alpha+\beta) / 2, \alpha} \\
& \times \int_{0}^{t-\varepsilon} \int_{\tau}^{t-\varepsilon}\|U(s, \tau)\|_{0,(\alpha+\beta) / 2}\|f(\tau, x(\tau), \dot{x}(\tau),(G x)(\tau),(H x)(\tau))\| d s d \tau \\
& \leq\left\|x_{0}-U(t, t-\varepsilon) x_{0}\right\|_{\alpha}+C(\beta, \alpha)\left\|x_{1}\right\|_{\beta} \varepsilon+C\left(\frac{\alpha+\beta}{2}, \alpha, \gamma_{1}\right) C\left(\beta, \frac{\alpha+\beta}{2}\right)\left\|x_{1}\right\|_{\beta} \varepsilon^{\gamma_{1}} \\
& +\frac{\omega C(\alpha, \gamma)}{1-\gamma}\|q\|_{L^{r}}\left(\frac{r-1}{r(2-\gamma)-1}\right)^{(r-1) / r} \varepsilon^{(r(2-\gamma)-1) / r} \\
& +\omega\|q\|_{L^{r}} C(\alpha, \gamma)\left(\frac{r-1}{r-r \gamma-1}\right)^{(r-1) / r} T^{(r-r \gamma-1) / r} \varepsilon \\
& +\omega\|q\|_{L^{r}} C\left(\frac{\alpha+\beta}{2}, \alpha, \gamma_{1}\right) C\left(\frac{\alpha+\beta}{2}, \gamma_{2}\right)\left(\frac{r-1}{r-1-r \gamma_{2}}\right)^{(r-1) / r} T^{\left(2 r-1-\gamma_{2}\right) / r} \mathcal{E}^{\gamma_{1}}
\end{aligned}
$$

where $0<\gamma_{1}<(\beta-\alpha) / 2,(\alpha+\beta) / 2<\gamma_{2}<1$, and

$$
\left\|\frac{d}{d t}(P x)(t)-\frac{d}{d t}\left(P_{\varepsilon} x\right)(t)\right\|_{\alpha} \leq \omega\|q\|_{L^{r}} C(\alpha, \gamma) \frac{r-1}{r-1-r \gamma} \varepsilon^{(r-1-r \gamma) /(r-1)}, \quad x \in \mathcal{X}
$$


This means that the set $\mathcal{K}(t)(\dot{\mathcal{K}}(t))$ can be approximated to an arbitrary degree of accuracy by a relatively compact set for $t \in\left(0, t_{1}\right]$. Hence $\mathcal{K}(t)(\dot{\mathcal{K}}(t))$ itself is relatively compact in $X_{\alpha}$ for $t \in\left(0, t_{1}\right]$. Define

$$
\begin{gathered}
\mathcal{K}\left(t_{1}+0\right)=\left\{\left.(P x)(t)\right|_{t=t_{1}}+J_{1}^{0}\left((P x)\left(t_{1}\right),\left.\frac{d}{d t}(P x)(t)\right|_{t=t_{1}}\right)\right\}, \\
\dot{\mathcal{K}}\left(t_{1}+0\right)=\left\{\left.\frac{d}{d t}(P x)(t)\right|_{t=t_{1}}+J_{1}^{1}\left((P x)\left(t_{1}\right),\left.\frac{d}{d t}(P x)(t)\right|_{t=t_{1}}\right)\right\} .
\end{gathered}
$$

Using relative compactness of $\mathcal{K}(t)$ and $\dot{\mathcal{K}}(t)\left(t \in\left[0, t_{1}\right]\right)$ and Assumption J, one can show that $\mathcal{K}\left(t_{1}+0\right)$ and $\dot{\mathcal{K}}\left(t_{1}+0\right)$ are relative compacts in $X_{\alpha}$. proved.

By the same procedure, the compactness of $\dot{\mathcal{K}}(t)$ and $\mathcal{K}(t)(t \in[0, T])$ can also be

Further, using representations of $(P x)(t)$ and $(d / d t)(P x)(t)$, properties of $\{\bar{U}(t, s), 0 \leq$ $s \leq t \leq T\}$ (see Theorem 2.1), and those above, one can show that $P x$ is piecewise equicontinuous. Summarily, $P$ is a compact operator in $\mathrm{PC}^{1}\left([0, T], \mathrm{X}_{\alpha}\right)$.

By virtue of Gronwall inequality (see Theorem 2.2), one can verify that $y=\{x \in$ $\left.\mathrm{PC}^{1}\left([0, T], \mathrm{X}_{\alpha}\right) \mid x=\delta P x, \delta \in[0,1]\right\}$ is a bounded subset of $\mathrm{PC}^{1}\left([0, T], \mathrm{X}_{\alpha}\right)$. According to Leray-Schauder fixed point theorem, $P$ has a fixed point in $\operatorname{PC}^{1}\left([0, T], X_{\alpha}\right)$. It can be given by the representation (3.1) and $x(t) \in D(A)$ for $t \in[0, T]$.

Remark 3.3. Similarly, under suitable assumptions, the following second-order nonlinear impulsive integro-differential equation of mixed type

$$
\begin{gathered}
\ddot{x}(t)+A(t) \dot{x}(t)=f(t, x(t), \dot{x}(t),(G x)(t),(H x)(t)), \quad t \in(0, T] \backslash(\Theta \cup \Lambda), \\
x(0)=x_{0}, \quad \Delta x\left(t_{i}\right)=J_{i}^{0}\left(x\left(t_{i}\right), \dot{x}\left(t_{i}\right)\right), \quad t_{i} \in \Theta, i=1,2, \ldots, n, \\
\dot{x}(0)=x_{1}, \quad \Delta \dot{x}\left(s_{j}\right)=J_{j}^{1}\left(x\left(s_{j}\right), \dot{x}\left(s_{j}\right)\right), \quad s_{j} \in \Lambda, j=1,2, \ldots, m,
\end{gathered}
$$

has a PC- $\alpha$-mild solution $x \in \mathrm{PC}^{1}\left([0, T], \mathrm{X}_{\alpha}\right)$ given by

$$
\begin{aligned}
x(t)= & x_{0}+\int_{0}^{t} U(\tau, 0) x_{1} d \tau+\int_{0}^{t} \int_{\tau}^{s} U(s, \tau) f(\tau, x(\tau), \dot{x}(\tau),(G x)(\tau),(H x)(\tau)) d s d \tau \\
& +\sum_{0<t_{i}<t} J_{i}^{0}\left(x\left(t_{i}\right), \dot{x}\left(t_{i}\right)\right)+\sum_{0<s_{j}<t} \int_{s_{j i}}^{t} U\left(s, s_{j}\right) J_{j}^{1}\left(x\left(s_{j}\right), \dot{x}\left(s_{j}\right)\right) d s .
\end{aligned}
$$

Remark 3.4. Assume that $A(0)$ has a compact resolvent, $\left(x_{0}, x_{1}\right) \in X_{\alpha} \times X_{\beta}, 0<\alpha<\beta \leq 1$, and $(\alpha+\beta) / 2<(r-1) / r$.

Under the Assumptions $\mathrm{A}$ and $\mathrm{F}$, the second-order nonlinear integro-differential equation of mixed type

$$
\begin{gathered}
\ddot{x}(t)+A(t) \dot{x}(t)=f(t, x(t), \dot{x}(t),(G x)(t),(H x)(t)), \quad t \in(0, T], \\
x(0)=x_{0}, \quad \dot{x}(0)=x_{1}
\end{gathered}
$$


has a unique $\alpha$-mild solution $x \in \mathrm{PC}^{1}\left([0, T], \mathrm{X}_{\alpha}\right)$ given by

$$
x(t)=x_{0}+\int_{0}^{t} U(\tau, 0) x_{1} d \tau+\int_{0}^{t} \int_{\tau}^{s} U(s, \tau) f(\tau, x(\tau), \dot{x}(\tau),(G x)(\tau),(H x)(\tau)) d s d \tau .
$$

\section{Existence of Optimal Controls}

We suppose that $Y$ is a reflexive Banach space from which the controls $u$ take the values. We denote a class of nonempty closed and convex subsets of $Y$ by $P_{f}(Y)$. Assume that the multifunction $\omega:[0, T] \rightarrow P_{f}(Y)$ is measurable and $\omega(\cdot) \subset E$ where $E$ is a bounded set of $Y$, the admissible control set $U_{\mathrm{ad}}=\left\{u \in L_{p}(E) \mid u(t) \in \omega(t)\right.$ a.e $\} \neq \emptyset$ (see [18, Proposition 1.7, page 142, Lemma 3.2, page 175]).

Consider the following controlled system:

$$
\begin{gathered}
\ddot{x}(t)+A(t) \dot{x}(t)=f(t, x(t), \dot{x}(t),(G x)(t),(H x)(t))+B(t) u(t), \quad t \in(0, T] \backslash \Theta, \\
x(0)=x_{0}, \quad \Delta x\left(t_{i}\right)=J_{i}^{0}\left(x\left(t_{i}\right), \dot{x}\left(t_{i}\right)\right), \quad t_{i} \in \Theta, i=1,2, \ldots, n, \\
\dot{x}(0)=x_{1}, \quad \Delta \dot{x}\left(t_{i}\right)=J_{i}^{1}\left(x\left(t_{i}\right), \dot{x}\left(t_{i}\right)\right), \quad t_{i} \in \Theta, i=1,2, \ldots, n .
\end{gathered}
$$

Assumption B. $B \in L_{\infty}([0, T], £(Y, X))$.

It is easy to see that $B u \in L_{p}([0, T], X)$ for all $u \in U_{\mathrm{ad}}$. Define $\mathcal{F}(t, x(t), \dot{x}(t),(G x)(t),(H x)(t))=f(t, x(t), \dot{x}(t),(G x)(t),(H x)(t))+B(t) u(t)$. It is obvious that $\mathcal{F}$ satisfies the Assumption $\mathrm{F}$.

Theorem 4.1. Suppose $A(0)$ has a compact resolvent. Under Assumptions $A, B, F$, and J, for every $u \in U_{a d}$, the system (4.1) has a PC- $\alpha$-mild solution corresponding to u provided $0<(\alpha+\beta) / 2, \beta<$ $\min \{(p-1) / p,(r-1) / r\}$.

Define $\tau=\left\{(x, u) \in \mathrm{PC}^{1}\left([0, T], X_{\alpha}\right) \times U_{\text {ad }} \mid x\right.$ as a PC- $\alpha$-mild solution of system (4.1) corresponding to the control $\left.u \in U_{\text {ad }}\right\} .(x, u) \in \tau$ is called a feasible pair. We consider the Lagrange problem $(\mathrm{P})$.

Find $\left(x^{0}, u^{0}\right) \in \tau$ such that

$$
J\left(x^{0}, u^{0}\right) \leq J(x, u), \quad \forall(x, u) \in \tau,
$$

where

$$
J(x, u)=\int_{0}^{T} l(t, x(t), \dot{x}(t), u(t)) d t
$$

We introduce some assumptions on $l$.

Assumption L. (1) The functional $l:[0, T] \times X_{\alpha} \times X_{\alpha} \times Y \rightarrow R \cup \infty$ is Borel measurable.

(2) $l(t, \cdot, \cdot, \cdot)$ is sequentially lower semicontinuous on $X_{\alpha} \times X_{\alpha} \times$ for almost all $t \in[0, T]$. 
(3) $l(t, x, y, \cdot)$ is convex on $Y$ for each $(x, y) \in X_{\alpha} \times X_{\alpha}$ and almost all $t \in[0, T]$.

(4) There exist constants $b \geq 0, c \geq 0, \varpi>0$, and $\varphi \in L_{1}([0, T], R)$ such that

$$
l(t, x, y, u) \geq \varphi(t)+b\|x\|_{\alpha}+c\|y\|_{\alpha}+\varpi\|u\|_{Y}^{p}, \quad \forall x, y \in X_{\alpha}, u \in Y .
$$

Now we can give the following result on the existence of optimal controls for problem $(\mathrm{P})$.

Theorem 4.2. Under assumptions of Theorem 4.1 and assumption $L$, the problem $(P)$ has a solution.

Proof. If $\inf \left\{J(x, u) \mid u \in U_{\text {ad }}\right\}=+\infty$, there is nothing to prove. Assume that $\inf \{J(x, u) \mid u \in$ $\left.U_{\mathrm{ad}}\right\}=m<+\infty$. By Assumption L, we have $m>-\infty$. By definition of infimum, there exists a sequence $\left\{u^{n}\right\} \subset U_{\text {ad }}$ such that $J\left(x^{u}, u^{n}\right) \rightarrow m$. Since $\left\{u^{n}\right\}$ is bounded in $L_{p}([0, T], Y)$, there exists a subsequence, relabeled as $\left\{u^{n}\right\}$, and $u^{0} \in L_{p}([0, T], Y)$ such that

$$
u^{n} \stackrel{w}{\longrightarrow} u^{0} \quad \text { in } L_{p}([0, T], Y) .
$$

Since $U_{\text {ad }}$ is closed and convex, from the Mazur Lemma, we have $u^{0} \in U_{\text {ad }}$.

Suppose $x^{n}$ is the PC- $\alpha$-mild solution of (4.1) corresponding to $u^{n}(n=0,1,2, \ldots)$. Then $x^{n}$ satisfies the following impulsive integral equation:

$$
\begin{aligned}
x^{n}(t)= & x_{0}+\int_{0}^{t} U(\tau, 0) x_{1} d \tau \\
& +\int_{0}^{t} \int_{0}^{s} U(s, \tau)\left[f\left(\tau, x^{n}(\tau), \dot{x}^{n}(\tau),\left(G x^{n}\right)(\tau),\left(H x^{n}\right)(\tau)\right)+B(\tau) u^{n}(\tau)\right] d \tau d s \\
& +\sum_{0<t_{i}<t} J_{i}^{0}\left(x^{n}\left(t_{i}\right), \dot{x}^{n}\left(t_{i}\right)\right)+\sum_{0<t_{i}<t} \int_{t_{i}}^{t} U\left(s, t_{i}\right) J_{i}^{1}\left(x^{n}\left(t_{i}\right), \dot{x}^{n}\left(t_{i}\right)\right) d s .
\end{aligned}
$$

Setting $F_{n}(t)=f\left(t, x^{n}(t), \dot{x}^{n}(t),\left(G x^{n}\right)(t),\left(H x^{n}\right)(t)\right)$, by Assumptions $\mathrm{F}(2)$ and $\mathrm{J}$, and Lemma 3.1, we obtain that $F_{n} \in \mathcal{L}_{r}([0, T], X)(r>1)$. Furthermore, there exists a subsequence, relabeled as $\left\{F_{n}\right\}$, and $\bar{F} \in L_{r}([0, T], X)$ such that $F_{n} \stackrel{w}{\rightarrow} \bar{F}$ in $L_{r}([0, T], X)$ as $n \rightarrow \infty$.

Define operators $Q: L_{r}([0, T], X) \rightarrow C([0, T], X)$, given by

$$
(Q y)(t)=\int_{0}^{t} U(t, s) y(s) d s
$$

By [7, Lemma 4.1], we have

$$
Q F_{n} \longrightarrow Q \bar{F} \text { in } C([0, T], X) \text { as } n \longrightarrow \infty \text {. }
$$


Consider the following impulsive differential equation:

$$
\begin{gathered}
\ddot{x}(t)+A(t) \dot{x}(t)=\bar{F}(t)+B(t) u^{0}(t), \quad t \in(0, T] \backslash \Theta, \\
x(0)=x_{0}, \quad \Delta x\left(t_{i}\right)=J_{i}^{0}\left(x\left(t_{i}\right), \dot{x}\left(t_{i}\right)\right), \quad t_{i} \in \Theta, i=1,2, \ldots, n, \\
\dot{x}(0)=x_{1}, \quad \Delta \dot{x}\left(t_{i}\right)=J_{i}^{1}\left(x\left(t_{i}\right), \dot{x}\left(t_{i}\right)\right), \quad t_{i} \in \Theta, i=1,2, \ldots, n, .
\end{gathered}
$$

By Theorem 3.2, we know that (4.9) have a PC- $\alpha$-mild solution

$$
\begin{aligned}
\bar{x}(t)= & x_{0}+\int_{0}^{t} U(\tau, 0) x_{1} d \tau+\int_{0}^{t} \int_{0}^{s} U(s, \tau)\left[\bar{F}(\tau)+B(\tau) u^{0}(\tau)\right] d \tau d s \\
& +\sum_{0<t_{i}<t} J_{i}^{0}\left(\bar{x}\left(t_{i}\right), \dot{\bar{x}}\left(t_{i}\right)\right)+\sum_{0<t_{i}<t} \int_{t_{i}}^{t} U\left(s, t_{i}\right) J_{i}^{1}\left(\bar{x}\left(t_{i}\right), \dot{\bar{x}}\left(t_{i}\right)\right) d s .
\end{aligned}
$$

Define

$$
\eta_{n}(t)=\int_{0}^{t} U(t, \tau)\left[F_{n}(\tau)-\bar{F}(\tau)+B(\tau) u^{n}(\tau)-B(\tau) u^{0}(\tau)\right] d \tau
$$

then, $\eta_{n} \rightarrow 0$ in $C\left([0, T], X_{\alpha}\right)$ as $n \rightarrow \infty$. Using Assumption J, we obtain

$$
\begin{aligned}
& \left\|x^{n}(t)-\bar{x}(t)\right\|_{\alpha}+\left\|\dot{x}^{n}(t)-\dot{\bar{x}}(t)\right\|_{\alpha} \\
& \quad \leq(T+1)\left\|\eta_{n}\right\|_{C\left([0, T], X_{\alpha}\right)}+b(T+1) \sum_{0<t_{i}<t}\left(C\left(\beta_{i}, \alpha\right)+1\right)\left(\left\|x^{n}\left(t_{i}\right)-\bar{x}\left(t_{i}\right)\right\|_{\alpha}+\left\|\dot{x}^{n}\left(t_{i}\right)-\dot{\bar{x}}\left(t_{i}\right)\right\|_{\alpha}\right) .
\end{aligned}
$$

Using the Gronwall lemma with impulse (see [17, Lemma 1.7.1]), we have

$$
\left\|x^{n}(t)-\bar{x}(t)\right\|_{\alpha}+\left\|\dot{x}^{n}(t)-\dot{\bar{x}}(t)\right\|_{\alpha} \leq b(T+1)^{2} \prod_{i=1}^{n}\left(C\left(\beta_{i}, \alpha\right)+1\right)\left\|\eta_{n}\right\|_{C([0, T], X)^{\prime}}
$$

that is, $x^{n} \rightarrow \bar{x}$ in $\operatorname{PC}^{1}\left([0, T], X_{\alpha}\right)$ as $n \rightarrow \infty$. Further,

$$
F_{n}(\cdot) \longrightarrow f(\cdot, \bar{x}(\cdot), \dot{\bar{x}}(\cdot),(G \bar{x})(\cdot),(H \bar{x})(\cdot)) \quad \text { in } L_{r}([0, T], X) \text { as } n \longrightarrow \infty
$$

By the uniqueness of limit, we have

$$
\bar{F}(t)=f(t, \bar{x}(t), \dot{\bar{x}}(t),(G \bar{x})(t),(H \bar{x})(t)),
$$


Furthermore,

$$
\begin{aligned}
\bar{x}(t)= & x_{0}+\int_{0}^{t} U(\tau, 0) x_{1} d \tau \\
& +\int_{0}^{t} \int_{0}^{s} U(s, \tau)\left[f(\tau, \bar{x}(\tau), \dot{\bar{x}}(\tau),(G \bar{x})(\tau),(H \bar{x})(\tau))+B(\tau) u^{0}(\tau)\right] d \tau d s \\
& +\sum_{0<t_{i}<t} J_{i}^{0}\left(\bar{x}\left(t_{i}\right), \dot{\bar{x}}\left(t_{i}\right)\right)+\sum_{0<t_{i}<t} \int_{t_{i}}^{t} U\left(s, t_{i}\right) J_{i}^{1}\left(\bar{x}\left(t_{i}\right), \dot{\bar{x}}\left(t_{i}\right)\right) d s
\end{aligned}
$$

Thus, $\bar{x}$ is a PC- $\alpha$-mild solution of (4.1) corresponding to $u^{0}$.

Since $\operatorname{PC}^{1}\left([0, T], X_{\alpha}\right) \hookrightarrow L_{1}\left([0, T], X_{\alpha}\right)$, using the Assumption L, we can obtain

$$
m=\lim _{n \rightarrow \infty} \int_{0}^{T} l\left(t, x^{n}(t), \dot{x}^{n}(t), u^{n}(t)\right) d t \geq \int_{0}^{T} l\left(t, \bar{x}(t), \dot{\bar{x}}(t), u^{0}(t)\right) d t=J\left(\bar{x}, u^{0}\right) \geq m
$$

This means that $J$ attains its minimum at $u^{0} \in U_{\text {ad }}$.

At the end of this section, an example is given to illustrate our theory. We consider the following problem:

$$
\begin{gathered}
\ddot{x}(t, y)+(t+1) \Delta \dot{x}(t, y) \\
=\dot{x}(t, y)+x(t, y)+\int_{0}^{t}(t-\tau) \sqrt{x^{2}(\tau, y)+\dot{x}^{2}(\tau, y)+1} d \tau \\
+\int_{0}^{1}(t+\tau)^{2} \sqrt{x^{2}(\tau, y)+\dot{x}^{2}(\tau, y)+1} d \tau \\
+u(t, y), \quad y \in \Omega, t \in(0,1] \backslash\left\{\frac{1}{10}, \frac{2}{10}, \ldots, \frac{9}{10}\right\}, \\
\left.x(t, y)\right|_{[0,1] \times \partial \Omega}=0, \quad x(0, y)=0,\left.\quad \frac{\partial}{\partial t} x(t, y)\right|_{[0,1] \times \partial \Omega}=0,\left.\quad \frac{\partial}{\partial t} x(t, y)\right|_{t=0}=0, \quad y \in \Omega, \\
x\left(\frac{i}{10}+0, y\right)-x\left(\frac{i}{10}-0, y\right)=\frac{1}{2} x\left(\frac{i}{10}-0, y\right), \quad i=1, \ldots, 9, \\
\dot{x}\left(\frac{i}{10}+0, y\right)-\dot{x}\left(\frac{i}{10}-0, y\right)=\frac{1}{2} \dot{x}\left(\frac{i}{10}-0, y\right), \quad i=1,2, \ldots, 9,
\end{gathered}
$$

where $\ddot{x}(t, y)=\left(\partial^{2} / \partial t^{2}\right) x(t, y), \dot{x}(t, y)=(\partial / \partial t) x(t, y), \Omega \subset R^{3}$ is bounded domain, and $\partial \Omega \in$ $C^{3}$.

Define $X=Y=L^{2}(\Omega), D(A(t))=H^{2}(\Omega) \cap H_{0}^{1}(\Omega)$, and $A(t) x=(t+1)\left(\partial^{2} x / \partial y_{1}^{2}+\right.$ $\left.\partial^{2} \mathrm{x} / \partial y_{2}^{2}+\partial^{2} x / \partial y_{3}^{2}\right)$ for $x \in D(A(t)) ; U_{\mathrm{ad}}$ is a nonempty bounded closed subset of $Y$. Let

$$
J(u)=\int_{0}^{1} \int_{\Omega}|x(t, \xi)|^{2} d \xi d t+\int_{0}^{1} \int_{\Omega}\left|\frac{\partial}{\partial t} x(t, \xi)\right|^{2} d \xi d t+\int_{0}^{1} \int_{\Omega}|u(t, \xi)|^{2} d \xi d t .
$$




$$
\begin{gathered}
\text { Define } V(\cdot)[u(\cdot)](y)=u(\cdot, y),[x(\cdot)](y)=x(\cdot, y),[(G x)(\cdot)](y)=\int_{0}(\cdot- \\
\tau) \sqrt{x^{2}(\tau, y)+\dot{x}^{2}(\tau, y)+1} d \tau,[(H x)(\cdot)](y)=\int_{0}^{1}(\cdot+\tau)^{2} \sqrt{x^{2}(\tau, y)+\dot{x}^{2}(\tau, y)+1} d \tau, \\
[F(\cdot, x(\cdot), \dot{x}(\cdot),(G x)(\cdot)),(H x)(\cdot))](y)=\int_{0}(\cdot-\tau) \sqrt{x^{2}(\tau, y)+\dot{x}^{2}(\tau, y)+1} d \tau+\int_{0}^{1}(\cdot+ \\
\tau)^{2} \sqrt{x^{2}(\tau, y)+\dot{x}^{2}(\tau, y)+1} d \tau+x(\cdot, y)+\dot{x}(\cdot, y),\left[g_{i}^{0}(x(i / 10))\right](y)=1 / 2 x(i / 10, y), \\
{\left[g_{i}^{1}(\dot{x}(i / 10))\right](y)=(1 / 2) \dot{x}(i / 10, y) ; \text { then } F \text { satisfies Assumption F. Thus the problem }} \\
(4.18) \text { can be rewritten as } \\
\ddot{x}(t)+A(t) \dot{x}(t)=F(t, x(t), \dot{x}(t),(G x)(t),(H x)(t))+V(t) u(t), \quad t \in(0,1] \backslash\left\{\frac{1}{10}, \ldots, \frac{9}{10}\right\}, \\
x(0)=0, \quad x\left(\frac{i}{10}+\right)-x\left(\frac{i}{10}-\right)=g_{i}^{0}\left(x\left(\frac{i}{10}\right)\right), \quad i=1,2, \ldots, 9, \\
\dot{x}(0)=0, \quad \dot{x}\left(\frac{i}{10}+\right)-\dot{x}\left(\frac{i}{10}-\right)=g_{i}^{1}\left(\dot{x}\left(\frac{i}{10}\right)\right), \quad i=1,2, \ldots, 9,
\end{gathered}
$$

with the cost function

$$
J(u)=\int_{0}^{1}\left(\|x(t)\|_{X}^{2}+\|\dot{x}(t)\|_{X}^{2}+\|u(t)\|_{X}^{2}\right) d t
$$

Obviously, satisfying all the assumptions given in our former Theorem 4.2, our results can be used in (4.20).

\section{Acknowledgments}

This work is supported by the National Natural Science Foundation of China under Grant no. 10961009, the Fok Ying Tung Education Foundation under Grant no. 121104 and Introducing Talents Foundation for the Doctor of Guizhou University.

\section{References}

[1] A. Pazy, Semigroups of Linear Operators and Applications to Partial Differential Equations, vol. 44 of Applied Mathematical Sciences, Springer, New York, NY, USA, 1983.

[2] H. Tanabe, "On the equations of evolution in a Banach space," Osaka Journal of Mathematics, vol. 12, pp. 363-376, 1960.

[3] H. Tanabe, Equations of Evolution, vol. 6 of Monographs and Studies in Mathematics, Pitman, Boston, Mass, USA, 1979.

[4] N. U. Ahmed, "Measure solutions for impulsive evolution equations with measurable vector fields," Journal of Mathematical Analysis and Applications, vol. 319, no. 1, pp. 74-93, 2006.

[5] Y. Peng, X. Xiang, and W. Wei, "Optimal feedback control for a class of strongly nonlinear impulsive evolution equations," Computers E Mathematics with Applications, vol. 52, no. 5, pp. 759-768, 2006.

[6] W. Wei, X. Xiang, and Y. Peng, "Nonlinear impulsive integro-differential equations of mixed type and optimal controls," Optimization, vol. 55, no. 1-2, pp. 141-156, 2006.

[7] X. Xiang, Y. Peng, and W. Wei, "A general class of nonlinear impulsive integral differential equations and optimal controls on Banach spaces," Discrete and Continuous Dynamical Systems. Series A, vol. 2005, pp. 911-919, 2005. 
[8] Y. Peng and X. Xiang, "Second order nonlinear impulsive time-variant systems with unbounded perturbation and optimal controls," Journal of Industrial and Management Optimization, vol. 4, no. 1, pp. 17-32, 2008.

[9] Y. Peng and X. Xiang, "Second-order nonlinear impulsive evolution equations with time-varying generating operators and optimal controls," Optimization, vol. 57, no. 6, pp. 827-840, 2008.

[10] Y. Peng, X. Xiang, and W. Wei, "Second-order nonlinear impulsive integro-differential equations of mixed type with time-varying generating operators and optimal controls on Banach spaces," Computers E Mathematics with Applications, vol. 57, no. 1, pp. 42-53, 2009.

[11] Y. Peng and X. Xiang, "Necessary conditions of optimality for second-order nonlinear impulsive integro-differential equations on Banach spaces," Nonlinear Analysis: Real World Applications, vol. 11, no. 4, pp. 3121-3130, 2010.

[12] R. P. Agarwal, M. Bohner, W.-S. Cheung, and S. R. Grace, "Oscillation criteria for first and second order forced difference equations with mixed nonlinearities," Mathematical and Computer Modelling, vol. 45, no. 7-8, pp. 965-973, 2007.

[13] E. Hernández M., M. Rabello, and H. R. Henríquez, "Existence of solutions for impulsive partial neutral functional differential equations," Journal of Mathematical Analysis and Applications, vol. 331, no. 2, pp. 1135-1158, 2007.

[14] L. Liu, Y. Wu, and X. Zhang, "On well-posedness of an initial value problem for nonlinear second-order impulsive integro-differential equations of Volterra type in Banach spaces," Journal of Mathematical Analysis and Applications, vol. 317, no. 2, pp. 634-649, 2006.

[15] H. Wang and H. Chen, "Boundary value problem for second-order impulsive functional differential equations," Applied Mathematics and Computation, vol. 191, no. 2, pp. 582-591, 2007.

[16] X. Zhang, L. Liu, and Y. Wu, "Global solutions of nonlinear second-order impulsive integrodifferential equations of mixed type in Banach spaces," Nonlinear Analysis: Theory, Methods $\mathcal{E}$ Applications, vol. 67, no. 8, pp. 2335-2349, 2007.

[17] T. Yang, Impulsive Control Theory, vol. 272 of Lecture Notes in Control and Information Sciences, Springer, Berlin, Germany, 2001.

[18] S. Hu and N. S. Papageorgiou, Handbook of Multivalued Analysis (Theory), vol. 419 of Mathematics and Its Applications, Kluwer Academic Publishers, Dordrecht, The Netherlands, 1997. 


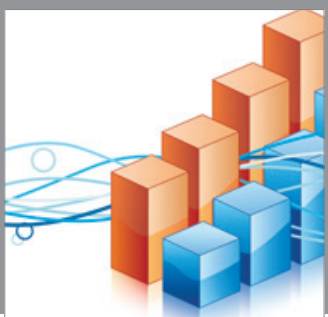

Advances in

Operations Research

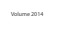

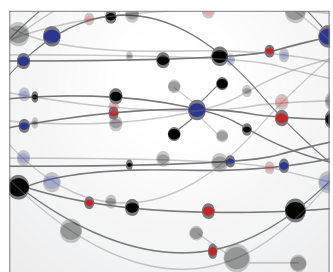

\section{The Scientific} World Journal
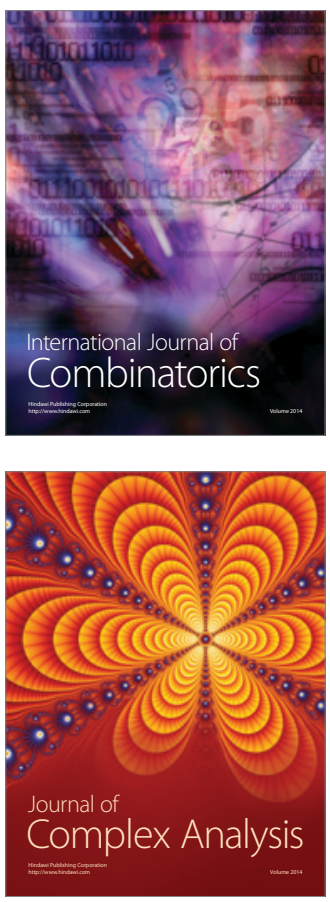

International Journal of

Mathematics and

Mathematical

Sciences
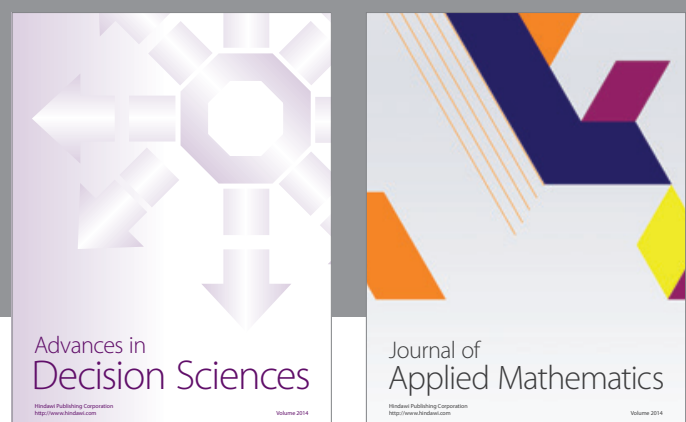

Journal of

Applied Mathematics
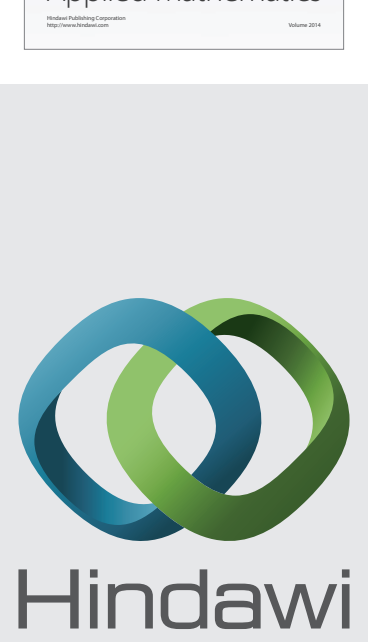

Submit your manuscripts at http://www.hindawi.com
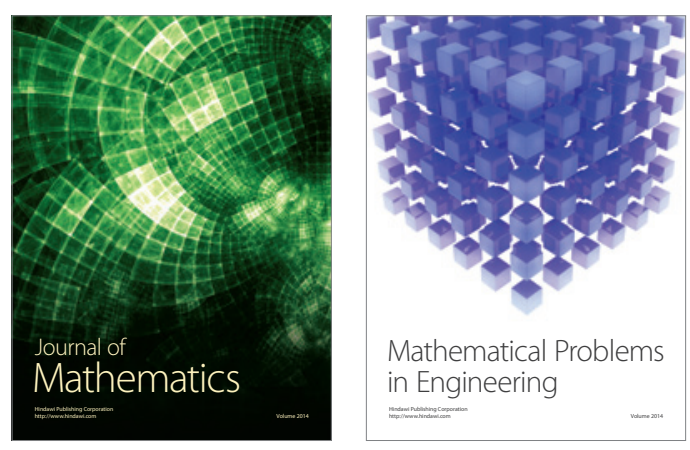

Mathematical Problems in Engineering
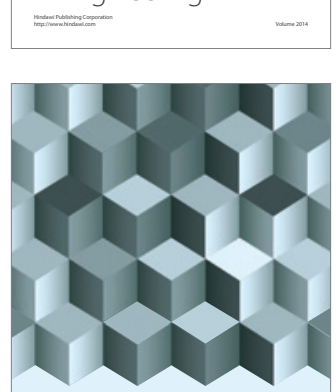

Journal of

Function Spaces
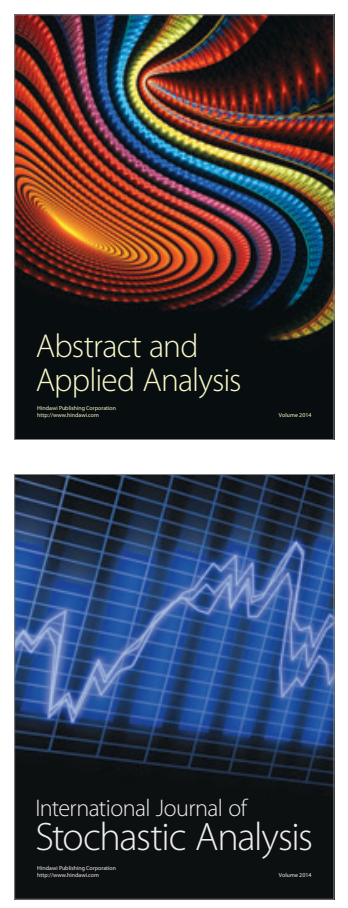

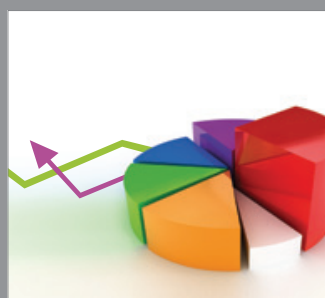

ournal of

Probability and Statistics

Promensencen
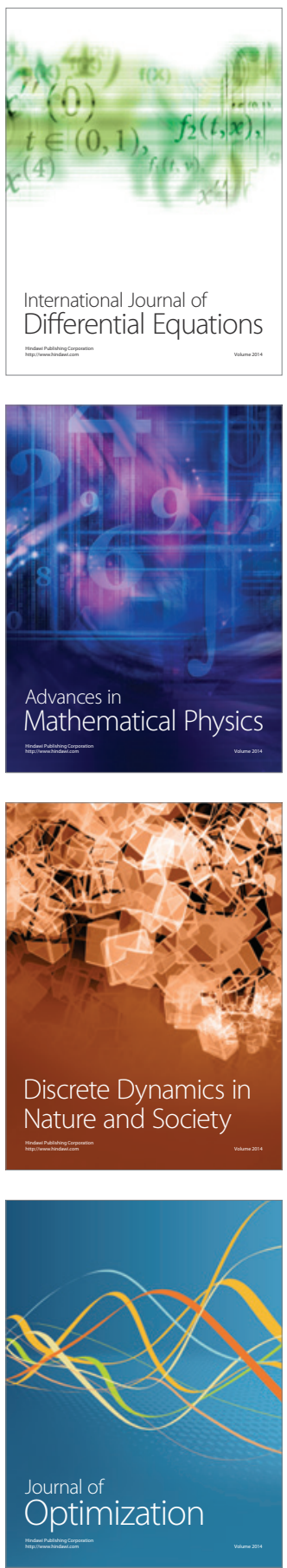